\title{
Epidemiología del asma en Colombia
}

\section{Epidemiology of asthma in Colombia}

PABlo Ramón GIL TORRES, MD. ${ }^{(1)}$

\section{Resumen}

Actualmente tenemos elementos de juicio que nos permiten evaluar la prevalencia del asma, su control y los factores asociados a su diagnóstico en Colombia.

En 1992 se publicó el primer estudio sobre prevalencia del asma en Cartagena incluyendo 4.000 sujetos, en el 2004 se obtuvo información por un estudio de corte transversal que además incluyó los diagnósticos de rinitis y dermatitis en seis ciudades de Colombia, en 2012 se publicó otro estudio que evaluó la prevalencia de síntomas de asma así como también rinitis y eczema, llama la atención en estos estudios que hay un porcentaje bajo de individuos con síntomas que reportan diagnóstico de asma por el médico, a pesar de incluso múltiples consultas a urgencias por esta causa.

En términos generales se observa una tendencia al aumento de la prevalencia del asma en Colombia en los últimos años especialmente en el grupo etario entre los 5 a 17 años y en los sujetos con atopia.

En el estudio PREPOCOL cuyo objetivo fue evaluar la prevalencia de enfermedad pulmonar obstructiva crónica en Colombia se encontró una prevalencia de 9\% de asma con un sub diagnóstico de $69,9 \%$

En cuanto a mortalidad hay un estudio publicado en 1998, los análisis del mismo reportan mortalidad por asma en Colombia más baja que la reportada en otros países latinoamericanos, pero se requiere de estudios más actualizados para evaluar la mortalidad por esta patología en Colombia.

Palabras clave: prevalencia de asma, mortalidad por asma, síntomas respiratorios.

\section{Abstract}

There is updated evidence that enables us to assess the prevalence of asthma in Colombia, and allows us to gain insight on factors associated with its diagnosis and control.

In 1992, the first study about the prevalence of asthma in the city of Cartagena was published. It included 4000 subjects. In 2004, information was brought about by a cross-cut study that also included the diagnoses of rhinitis and dermatitis in six cities of Colombia. In 2012, a study was published that assessed the prevalence of symptoms of asthma, as well as rhinitis and eczema. In these studies, it is noteworthy that a low percentage of individuals with symptoms report having been diagnosed with asthma by a physician, even despite multiple visits to the emergency ward for this reason.

In general terms, a tendency to an increase in the prevalence of asthma has been observed over the past few years, especially in the 5-17 years age group and in subjects with atopy.

In the PREPOCOL study, whose objective was to assess the prevalence of chronic obstructive pulmonary disease in Colombia, a $9 \%$ prevalence of asthma was found, with a $69,9 \%$ rate of underdiagnosis.

With regard to mortality, there is a study published in 1998 whose analyses report that asthma mortality rates in Colombia are lower than those reported in other Latin American countries. However, updated studies are required to assess the mortality of asthma in Colombia.

Keywords: prevalence of asthma, mortality of asthma, respiratory symptoms.

(1)Médico internista, Departamento de Medicina Interna, Hospital Universitario San Ignacio. Bogotá, Colombia .

Correspondencia: Pablo Ramón Gil Torres, correo electrónico: pablogil@outlook.com Recibido: 01/05/15. Aceptado: 15/05/15. 


\section{Introducción}

Respecto a la epidemiología del Asma en Colombia en adultos, se han publicado cuatro estudios que aportan información que permite tener una visión muy completa de la prevalencia de la enfermedad, su control y los factores asociados a su diagnóstico.

\section{Estudios publicados}

La epidemiología del asma en Colombia comenzó a estudiarse por Caraballo et al. en 1992, cuando se publicó el primer estudio sobre prevalencia en Cartagena (1). Se entrevistaron 4.000 sujetos considerándose como asmáticos aquellos que consultaron al médico en el último año debido a disnea acompañada de sibilancias y recibían medicamentos para el asma. Se observó una prevalencia acumulada del $8,8 \%$, donde un $60 \%$ correspondió a los pacientes menores de 15 años. Además se documentó que un $45 \%$ de los individuos tenían historia familiar de asma (1).

Posteriormente, en 2004, se obtuvo información sobre la prevalencia del asma en otras regiones del país. Dennis et al. llevaron a cabo un estudio de corte transversal para determinar la prevalencia del asma, la rinitis y la dermatitis en seis ciudades colombianas, mediante el empleo de un cuestionario estandarizado y pruebas de laboratorio para determinar la presencia de atopia (2). Se recolectó información de 6.507 sujetos con edad menor a 59 años entre los años 1998 y 2000, en Bogotá, Cali, Barranquilla, Medellín, San Andrés y Bucaramanga, documentándose una prevalencia de asma del 10,4\%, siendo estas últimas tres ciudades las de las cifras más altas. De estos pacientes, un $76 \%$ se consideró atópico con base en niveles altos de IgE total o la presencia de IgE específica contra al menos un alergeno.

A pesar de la prevalencia documentada, solo el $6,2 \%$ de los sujetos encuestados reportó haber sido diagnosticado con asma por su médico, cifra que indicaría un posible subdiagnóstico (2). Además, se evidenció que un $38 \%$ de los sujetos asmáticos había consultado al servicio de urgencias o había sido hospitalizado por esta causa. Finalmente, era preocupante que un $48,8 \%$ de los encuestados reportaron haber perdido al menos un día de trabajo o escuela en los últimos seis meses debido al asma, evidenciándose así la alta carga económica de esta enfermedad.

Con el fin de evaluar la variación epidemiológica del asma en Colombia se publicó otro estudio en 2012 que nuevamente evaluó la prevalencia de síntomas de asma, así como del eczema atópico y la rinitis en las mismas seis ciudades evaluadas en el estudio anterior, en menores de 59 años (3). Entre los años 2009 y 2010 se practicó el mismo cuestionario empleado previamente en 5.978 personas y se obtuvo una prevalencia del $12 \%$. Bucaramanga y Cali fueron las ciudades con mayor prevalencia de síntomas de asma reportada durante toda la vida. Un $60 \%$ de los encuestados fueron clasificados como atópicos con base en las pruebas descritas.

Al igual que en el estudio previo, solo un $7 \%$ de los encuestados reportó haber sido diagnosticado con asma por algún médico y un $43 \%$ afirmó haber requerido consultar al servicio de urgencias u hospitalización en el último año (3). Un 55\% de los sujetos con asma reportaron haber perdido algún día de trabajo o estudio en los últimos seis meses, con una mediana de 4 días, hecho que reafirma la gran carga económica de la enfermedad para el país.

Pese a que los autores principales de estos estudios fueron los mismos y se usó una metodología similar, las diferencias en los grupos de edades de los sujetos, los métodos y el análisis de la información, así como los alérgenos empleados para determinar la presencia de atopia precluyen la posibilidad de una comparación formal entre los mismos. No obstante, se puede observar una tendencia al aumento de la prevalencia del asma en Colombia en los últimos años, principalmente en el grupo etario entre los 5 y 17 años, y adicionalmente, se evidencia una prevalencia persistentemente alta de sujetos con atopia. Por otra parte, la severidad de la enfermedad permanece alta, lo cual puede ser secundario a subdiagnóstico. Esto lleva a pensar que hay un acceso limitado a la valoración por medicina especializada, lo que da lugar a un control subóptimo de la enfermedad. El seguimiento inapropiado del asma también contribuye a la severidad del cuadro y al alto empleo de los servicios de salud. 
Por otra parte, en 2015 se publicó un estudio de corte transversal, basado en la población, con el fin de evaluar la prevalencia, el subdiagnóstico y los factores de riesgo para asma en sujetos mayores de 40 años de edad (4). En el trabajo, 5.539 individuos entre los 40 y 93 años de edad de Barranquilla, Bucaramanga, Cali, Medellín y Bogotá fueron encuestados para evaluar los síntomas respiratorios y factores de riesgo para la enfermedad. Además se les practicó una espirometría para confirmar el diagnóstico.

Esta información fue extraída del estudio PREPOCOL, cuyo objetivo principal fue la evaluación de la prevalencia de la enfermedad pulmonar obstructiva crónica en Colombia (5). Se encontró una prevalencia del 9\% de asma en este grupo poblacional, con un subdiagnóstico importante del $69,9 \%$, mayor en los sujetos de 64 o más años de edad (79\%). Las ciudades con más alta prevalencia fueron Bogotá y Medellín, encontrándose otros factores de riesgo como: género femenino, familiar en primer grado con asma, haber padecido una enfermedad respiratoria antes de los 16 años de edad, obesidad, bajo nivel educativo, exposición al humo de leña y exposición ocupacional a las partículas del polvo, gases o vapores. Estos datos demuestran una prevalencia similar a los estudios anteriores y preocupa el alto grado de subdiagnóstico que puede deberse a una menor percepción de los síntomas en este grupo etario.

Finalmente, en cuanto a mortalidad, solo se ha publicado un estudio en 1998 en el que se evaluaron las tasas entre 1979 y 1994 (6). Mediante la revisión de los certificados de defunción del Departamento Nacional de Estadísticas (DANE), se documentó una tendencia al descenso de la mortalidad por asma al pasar de 2,06 por cada 100.000 habitantes en 1979 a 1,6 en 1994. Las muertes por asma fueron más frecuentes en sujetos mayores de 35 años comparados con menores de 5 años o sujetos entre los 5 y 34 años. No se observó una variación significativa en la mortalidad entre hombres y mujeres. Un $62 \%$ de las muertes por asma ocurrieron en el hogar, $31 \%$ en los hospitales y $6,7 \%$ en otros lugares. En la mayoría de los casos las muertes se dieron en áreas urbanas, con un $73 \%$. Estos resultados demuestran que la mortalidad por asma en Colombia es más baja que la reportada en otros países latinoamericanos como Costa Rica, Argentina, Cuba, Uruguay y Venezuela, pero más alta que en Chile (7). La mejoría en esta tasa puede obedecer a las políticas de salud pública implementadas en años recientes en el país para mejorar la atención en salud, en particular en cuanto a esta enfermedad. No obstante, se requieren estudios actualizados respecto a mortalidad por esta patología en Colombia.

\section{Conflictos de interés}

El autor declara no tener conflictos de interés.

\section{Bibliografía}

1. Caraballo L, Cadavid A, Mendoza J. Prevalence of asthma in a tropical city of Colombia. Ann Allergy. 1992;68(6):525-9.

2. Dennis R, Caraballo L, García E, Caballero A, Aristizábal G, Córdoba $\mathrm{H}$, et al. Asthma and other allergic conditions in Colombia: a study in 6 cities. Ann Allergy Asthma Immunol Off Publ Am Coll Allergy Asthma Immunol. 2004;93(6):568-74.

3. Dennis RJ, Caraballo L, García E, Rojas MX, Rondon MA, Pérez A, et al. Prevalence of asthma and other allergic conditions in Colombia 2009-2010: a cross-sectional study. BMC Pulm Med. 2012;12:17.

4. Gonzalez-Garcia M, Caballero A, Jaramillo C, Maldonado D, Torres-Duque CA. Prevalence, risk factors and underdiagnosis of asthma and wheezing in adults 40 years and older: A population-based study. J Asthma Off J Assoc Care Asthma. 2015;52(8):823-30.

5. Caballero A, Torres-Duque CA, Jaramillo C, Bolívar F, Sanabria F, Osorio P, et al. Prevalence of COPD in five Colombian cities situated at low, medium, and high altitude (PREPOCOL study). Chest. 2008;133(2):343-9.

6. Vergara C, Caraballo L. Asthma mortality in Columbia. Ann Allergy Asthma Immunol Off Publ Am Coll Allergy Asthma Immunol. 998;80(1):55-60.

7. Neffen H, Baena-Cagnani CE, Malka S, Solé D, Sepúlveda R, Caraballo L, et al. Asthma mortality in Latin America. J Investig Allergol Clin Immunol. 1997;7(4):249-53. 